\title{
Study of the sub-AU disk of the Herbig B[e] star HD 85567 with near-infrared interferometry ${ }^{\star} \star \star \star$
}

\author{
J. Vural ${ }^{1, \star \star \star}$, S. Kraus ${ }^{2}$, A. Kreplin ${ }^{1}$, G. Weigelt ${ }^{1}$, E. Fossat ${ }^{3}$, F. Massi ${ }^{4}$, K. Perraut ${ }^{5}$, and F. Vakili ${ }^{3}$ \\ ${ }^{1}$ Max-Planck-Institut für Radioastronomie, Auf dem Hügel 69, 53121 Bonn, Germany \\ e-mail: jvural@mpifr-bonn.mpg.de \\ 2 University of Exeter, School of Physics, Physics Building, Stocker Road, Exeter, EX4 4QL, UK \\ ${ }^{3}$ Laboratoire Lagrange, UMR7293, Université de Nice Sophia-Antipolis, CNRS, Observatoire de la Côte d'Azur, 06300 Nice, France \\ 4 INAF - Osservatorio Astrofisico di Arcetri, Largo E. Fermi 5, 50125 Firenze, Italy \\ 5 UJF-Grenoble 1/CNRS-INSU, Institut de Planétologie et d'Astrophysique de Grenoble (IPAG) UMR 5274, \\ 38041 Grenoble, France
}

Received 15 May 2014 / Accepted 10 July 2014

\section{ABSTRACT}

\begin{abstract}
Context. The structure of the inner disk of Herbig Be stars is not well understood. The continuum disks of several Herbig Be stars have inner radii that are smaller than predicted by models of irradiated disks with optically thin holes.

Aims. We study the size of the inner disk of the Herbig B[e] star HD 85567 and compare the model radii with the radius suggested by the size-luminosity relation.

Methods. The object was observed with the AMBER instrument of the Very Large Telescope Interferometer. We obtained $K$-band visibilities and closure phases. These measurements are interpreted with geometric models and temperature-gradient models.

Results. Using several types of geometric star-disk and star-disk-halo models, we derived inner ring-fit radii in the $K$ band that are in the range of 0.8-1.6 AU. Additional temperature-gradient modeling resulted in an extended disk with an inner radius of $0.67_{-0.21}^{+0.51} \mathrm{AU}$, a high inner temperature of $2200_{-350}^{+750} \mathrm{~K}$, and a disk inclination of $53_{-11}^{+15 \circ}$.

Conclusions. The derived geometric ring-fit radii are approximately 3-5 times smaller than that predicted by the size-luminosity relation. The small geometric and temperature-gradient radii suggest optically thick gaseous material that absorbs stellar radiation inside the dust disk.
\end{abstract}

Key words. stars: individual: HD 85567 - stars: pre-main sequence - stars: formation - circumstellar matter techniques: interferometric

\section{Introduction}

Infrared interferometric observations suggest that the circumstellar environment of Herbig $\mathrm{Be}(\mathrm{HBe})$ and Herbig Ae (HAe) stars are significantly different. In contrast to the lower-mass HAe stars, the $K$-band continuum radii of several HBe stars are significantly smaller than predicted by the size-luminosity relation (Monnier et al. 2005; Eisner et al. 2004; Kraus et al. 2008b; Weigelt et al. 2011; Kreplin et al. 2012). These smaller radii can be explained by the presence of an optically thick gas inside the dust disk. This optically thick gas can absorb the stellar ultraviolet (UV) radiation and allows dust to exist closer to the star (Monnier \& Millan-Gabet 2002).

The object HD 85567 (V596 Car, Hen 3-331) is a B-type star at a distance of $1.5 \pm 0.5 \mathrm{kpc}$ (see stellar parameters in Table 1). The evolutionary status of HD 85567 is not yet wellestablished. Miroshnichenko et al. (2001) suggested the object to be a main-sequence $\mathrm{B}[\mathrm{e}]$ star. Other studies reported that HD 85567 is a young stellar object (Lamers et al. 1998;

^ Based on observations made with ESO telescopes at the La Silla Paranal Observatory under program IDs 080.C-0541(C), 082.C0893(A), 084.C-0848(B).

$\star \star$ Appendix $\mathrm{A}$ is available in electronic form at

http: //wwW . aanda. org

$\star \star \star$ Member of the International Max Planck Research School (IMPRS) for Astronomy and Astrophysics at the Universities of Bonn and Cologne.
Table 1. Stellar parameters of HD 85567.

\begin{tabular}{rc}
\hline \hline Parameter & Value \\
\hline Spectral type & ${\mathrm{B} 2 \mathrm{~V}^{a}}^{a}$ \\
Distance $[\mathrm{kpc}]$ & $1.5 \pm 0.5^{a}$ \\
$M_{*}\left[M_{\odot}\right]$ & $12 \pm 2^{b}$ \\
$\log \left(T_{*}[\mathrm{~K}]\right)$ & $4.32 \pm 0.08^{b}$ \\
$\log \left(L_{*}\left[L_{\odot}\right]\right)$ & $4.17 \pm 0.16^{b}$ \\
$R_{*}\left[R_{\odot}\right]$ & $9 \pm 2^{b}$ \\
$A_{\mathrm{V}}$ & $1.1 \pm 0.1^{b}$ \\
$\dot{M}\left[10^{-6} M_{\odot} \mathrm{yr}^{-1}\right]$ & $6.3_{-2}^{+4 c}$ \\
\hline &
\end{tabular}

References. ${ }^{(a)}$ Miroshnichenko et al. (2001); ${ }^{(b)}$ Verhoeff et al. (2012); (c) Ilee (2013).

Malfait et al. 1998; Verhoeff et al. 2012; Wheelwright et al. 2013). Miroshnichenko et al. (2001) proposed the existence of a close binary companion that can interact with the circumstellar disk of HD 85567. Binarity is believed to be a key property in B [e] stars (Miroshnichenko 2007). Baines et al. (2006) detected a binary companion with a separation of $\gtrsim 500$ mas.

We use our near-infrared (NIR) interferometric observations to investigate the inner disk structure of HD 85567. The paper is organized as follows. We describe the observations and the data reduction in Sect. 2. The modeling is presented in Sect. 3 and the results are discussed in Sect. 4. 


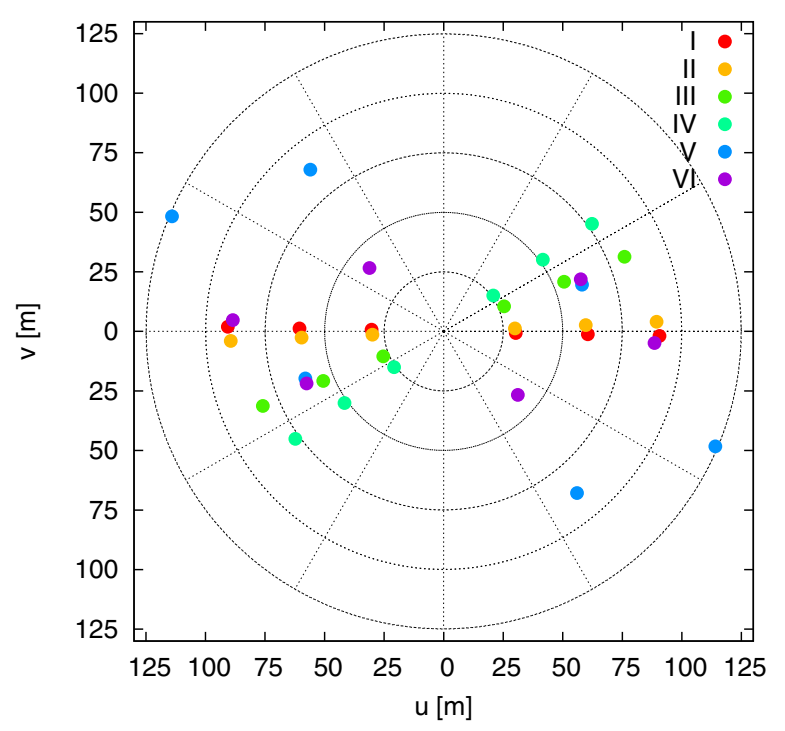

Fig. 1. The $u v$ coverage of our AMBER measurements of HD 85567 (see Table 2).

\section{Observation and data reduction}

The observations of HD 85567 were carried out using the NIR three-beam combiner VLTI/AMBER (Petrov et al. 2007) in three different nights. We obtained a total of six measurements in the low spectral resolution mode $(R=30$; Fig. 1 , Table 2$)$. The data were reduced with amdlib 3.0.5 (Tatulli et al. 2007; Chelli et al. 2009). We applied a signal-to-noise frame selection (20\% of the highest fringe signal-to-noise ratio (S/N); Tatulli et al. 2007) to the raw files of object and calibrator to obtain an improved visibility calibration. An additional improvement of the visibility calibration was achieved by equalizing the optical path difference (OPD) histograms of the object and calibrator (Kreplin et al. 2012) to account for atmospheric OPD drifts.

We derived $K$-band closure phases (Fig. 2) and visibilities (Fig. 3). The closure phases are zero within the error bars, indicating that the brightness distribution of our source is centrosymmetric. Except in measurement VI, amdlib 3.0.5 did not compute visibilities and closure phases for wavelengths in the range of approximately $2.0-2.1 \mu \mathrm{m}$ because of low $\mathrm{S} / \mathrm{N}$. The $\mathrm{S} / \mathrm{N}$ of the $H$-band data was also too low to derive $H$-band visibilities and closure phases.

The spectral energy distribution (SED; see Fig. 4) was reconstructed from dereddened values found in the literature (Verhoeff et al. 2012) and data from the Spitzer Space Telescope (IRS, program ID: 3470 ).

\section{Modeling}

\subsection{Geometric modeling}

The NIR emission of Herbig stars is believed to originate mainly in a ring-like region at the inner edge of the disk, which is often associated with a puffed-up inner rim (Natta et al. 2001; Dullemond et al. 2001). Thus, the size of the NIR emission region is often approximated with a geometric ring model, and the ring-fit radius can be compared to theoretical predictions (Millan-Gabet et al. 2001; Monnier \& Millan-Gabet 2002; Eisner et al. 2003; Dullemond \& Monnier 2010).

\footnotetext{
1 http://www.jmmc.fr/data_processing_amber.htm
}

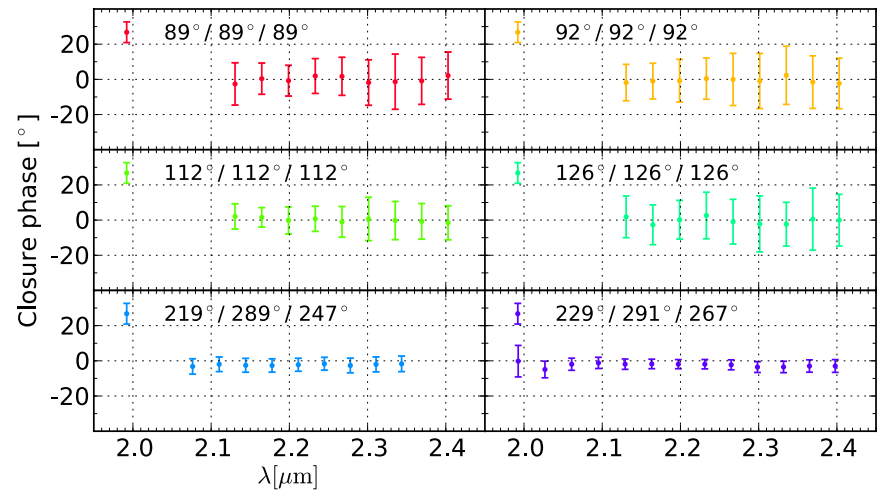

Fig. 2. Derived closure phases. The color coding is the same as in Fig. 1.

Here, we apply several different ring models, all containing an unresolved stellar component and a ring with a width of $20 \%$ of the inner ring radius (Monnier et al. 2005). We also investigate inclination effects (i.e., elongated rings) and the influence of an extended halo (see Table 3 ).

The variables of the two-dimensional total visibility $|V|=$ $\left|V\left(P A, v_{\mathrm{uv}}\right)\right|$ are the position angle (PA) of the measurement and the spatial frequency $v_{\mathrm{uv}}=B_{\text {proj }} / \lambda$ (see Table 2$)$. It can be described by

$|V|=\left|\left(1-f_{*}-f_{\text {halo }}\right) V_{\text {ring }}+f_{*} V_{*}+f_{\text {halo }} V_{\text {halo }}\right|$,

where $V_{*}=1$ is the visibility of the star, $V_{\text {ring }}$ is the visibility of the ring-shaped disk, $V_{\text {halo }}$ is the visibility of the halo, $f_{\text {halo }}$ is the flux contribution of the halo, and $1-f_{*}-f_{\text {halo }}$ is the flux contribution of the ring-like disk. The wavelength-dependent relative flux contribution $f_{*}$ of the stellar component to the total emission was derived from the SED (Fig. 4). The model parameters are the inner ring radius $R_{\text {in }}$ (semi-major axis in the elongated case) of the extended ring, the inclination $i$, the position angle $\vartheta$ of the semimajor axis of the elongated ring, and $f_{\text {halo }}$. An overview of our fit models, their parameters, and the resulting $\chi_{\text {red }}^{2}$ is presented in Table 3. We obtain inner radii between $0.78 \mathrm{AU}$ ( $\sim 0.5$ mas) and $1.55 \mathrm{AU}(\sim 1.0 \mathrm{mas})$ and inclination angles in the range of $\sim 56^{\circ}$ to $\sim 70^{\circ}$. The large error bars of the NIR visibilities do not allow us to make a definite decision on the elongation and the orientation of the disk as models without elongation fit the data as well (see Table 3).

\subsection{Temperature-gradient model}

To interpret our data, we model our visibility measurements and the literature SED of HD 85567 with a temperature-gradient model. This kind of modeling has already been used for modeling AMBER data from young stars of different types by, for example, Malbet et al. (2005), Kraus et al. (2010, 2012), Kreplin et al. (2012), Chen et al. (2012), and Vural et al. (2014).

The temperature distribution $T(r)$ of a circumstellar disk is assumed to be: $T(r)=T_{\text {in }} \cdot\left(r / r_{\text {in }}\right)^{-q}$, where $r$ is the radius, $q$ is the temperature power-law index, and $T_{\text {in }}$ the temperature at the inner disk radius $r_{\text {in. }}$. The disk is modeled as the sum of a large number of narrow rings; each has a temperature $T(r)$. We integrate the blackbody spectra of the rings and the intensity distribution of these rings to obtain the total energy distribution and the visibilities. We allow the model disk to be inclined, thus introducing the inclination $i\left(i=0^{\circ}\right.$ if the disk is face-on) and the position angle $\vartheta$ (measured east of north) of the semi-major axis of the model disk as additional parameters. 
Table 2. AMBER observation log.

\begin{tabular}{rrrrrrrrr}
\hline \hline$\#$ & Night & Configuration & $\begin{array}{r}B_{\text {proj }} \\
{[\mathrm{m}]}\end{array}$ & $\begin{array}{r}\text { PA } \\
{\left[{ }^{\circ}\right]}\end{array}$ & $\begin{array}{r}\text { Seeing } \\
{\left[{ }^{\prime}\right]}\end{array}$ & $\begin{array}{r}\text { DIT } \\
{[\mathrm{ms}]}\end{array}$ & $\begin{array}{r}\text { Calibrator } \\
\text { Calibrator diameter } \\
{[\mathrm{mas}]}\end{array}$ \\
\hline I & $2008-02-22$ & A0-D0-H0 & $60 / 91 / 30$ & $89 / 89 / 89$ & 0.65 & 21 & HD 50281 & $\begin{array}{r}1.0 \pm 0.1^{a} \\
\text { II }\end{array}$ \\
\hline $2008-02-22$ & A0-D0-H0 & $60 / 91 / 30$ & $92 / 92 / 92$ & 0.72 & 100 & HD 85313 & $0.451 \pm 0.045^{b}$ \\
III & $2008-02-22$ & A0-D0-H0 & $55 / 82 / 27$ & $112 / 112 / 112$ & 0.69 & 100 & HD 85313 & $0.451 \pm 0.045^{b}$ \\
IV & $2008-02-22$ & A0-D0-H0 & $51 / 77 / 26$ & $126 / 126 / 126$ & 0.57 & 100 & HD 85313 & $0.451 \pm 0.045^{b}$ \\
V & $2008-12-14$ & U1-U3-U4 & $88 / 61 / 124$ & $219 / 289 / 247$ & - & 26 & HD 50277 & $0.356 \pm 0.036^{b}$ \\
VI & $2009-12-31$ & U2-U3-U4 & $41 / 62 / 89$ & $229 / 291 / 267$ & 1.1 & 21 & HD 85313 & $0.451 \pm 0.045^{b}$ \\
\hline
\end{tabular}

Notes. The resulting $u v$ coverage is shown in Fig. $1 . B_{\text {proj }}$ describes the projected baseline length, PA the position angles of the baselines, and DIT the detector integration time. We assumed the calibrator diameter error to be $10 \%$.

References. ${ }^{(a)}$ Pasinetti Fracassini et al. (2001), ${ }^{(b)}$ Lafrasse et al. (2010).

Table 3. Overview of the geometric models.

\begin{tabular}{|c|c|c|c|c|c|c|}
\hline Model & $\begin{array}{r}R_{\text {in }} \\
{[\mathrm{AU}]}\end{array}$ & $\begin{array}{r}R_{\min } \\
{[\mathrm{AU}]}\end{array}$ & $\begin{array}{l}i \\
\circ\end{array}$ & $\vartheta$ & $f_{\text {halo }}$ & $\chi_{\text {red }}^{2}$ \\
\hline Ring & $1.08 \pm 0.38$ & $1.08 \pm 0.38$ & $\ldots$ & $\ldots$ & $\ldots$ & 2.69 \\
\hline Ring + extended halo & $0.78 \pm 0.31$ & $0.78 \pm 0.31$ & $\ldots$ & $\ldots$ & $0.09 \pm 0.01$ & 0.39 \\
\hline Elongated ring & $1.55 \pm 0.53$ & $0.53 \pm 0.20$ & $70.0 \pm 0.01$ & $155.7 \pm 2.1$ & $\ldots$ & 1.88 \\
\hline Elong. ring + extended halo & $1.07 \pm 0.43$ & $0.60 \pm 0.32$ & $55.8 \pm 0.09$ & $162.2 \pm 5.5$ & $0.09 \pm 0.01$ & 0.74 \\
\hline
\end{tabular}

Notes. All models consist of the above ring-like disks, halos, and an unresolved stellar source. The distance uncertainty (Table 1) is taken into account in the above uncertainties for the radii. The radii $R_{\mathrm{in}}$ and $R_{\min }$ are the semi-major and the semi-minor axis of the ring models, respectively. The variable $\vartheta$ is the position angle of the semi-major axis.

Table 4. Scanned parameter space and best-fit temperature-gradient model described in Sect. 3.2.

\begin{tabular}{llll}
\hline \hline Parameter & Scan range & $N_{\text {val }}$ & Best-fit value \\
\hline$r_{\text {in }}[\mathrm{AU}]$ & $0.04-10$ & 40 & $0.67_{-0.15}^{+0.51}$ \\
$\Delta r[\mathrm{AU}]$ & $0.01-50$ & 30 & $24.8_{-8.5}^{+20.5}$ \\
$T_{\text {in }}[\mathrm{K}]$ & $200-8000$ & 40 & $2200_{-341}^{+752}$ \\
$q$ & $0.2,0.5,0.75,1.0$ & 4 & 0.75 \\
$i\left[^{\circ}\right]$ & $0-90$ & 30 & $52.6_{-11.0}^{+14.7}$ \\
$\vartheta\left[^{\circ}\right]$ & $0-170$ & 30 & $121.4_{-46.8}^{30.6+}$ \\
\hline
\end{tabular}

Notes. $N_{\text {val }}$ is the number of parameter values used. The spacing between the single parameter values is linear or as indicated, except for $r_{\text {in }}$, where the spacing is logarithmic. For example, the parameter values for $r_{\text {in }}$ are $0.10 \mathrm{AU}, 0.11 \mathrm{AU}, 0.13 \mathrm{AU}, \ldots, 10 \mathrm{AU}$.

Our model includes a stellar component with the parameters from Table 1 (distance, $T_{*}, R_{*}$, and $L_{*}$ ) and a surrounding circumstellar temperature-gradient disk extending to an outer radius of $r_{\text {out }}=r_{\text {in }}+\Delta r$. Each computed model contains the visibilities for all baseline-position angle combinations in our measurements. We calculated the models for all combinations of the parameter values as described in Table 4, where each parameter was varied in 4 to 40 steps within the described scan ranges. This method required the calculation of approximately 170 million models.

To find the best-fit temperature-gradient model, we calculated the $\chi_{\text {red }}^{2}$ (using $\chi^{2}=\chi_{\mathrm{SED}}^{2}+\chi_{\mathrm{Vis}}^{2}$ ) of each model and obtained a best-fit model with $\chi_{\text {red }}^{2} \sim 1.0$ (see Fig. A.1 in the Appendix). The parameters of the best-fit model are listed in Table 4 and will be discussed in the following section. The uncertainties are $3 \sigma$ errors. The model curves are shown in Figs. 3 and 4.

\section{Discussion}

From our geometric modeling, we obtained different ringfit radii for different star-disk and star-disk-halo models (see Table 3). We achieve significantly better $\chi_{\text {red }}^{2}$ if the model has a halo component. The $\chi_{\text {red }}^{2}$ closest to one was obtained for the geometric ring model of an elongated ring and a halo. The inner ring radius of this model is $R_{\text {in }} \sim 1.1 \pm 0.4$ AU. Wheelwright et al. (2013) estimated the ring radius with a symmetric star-ring model including resolved background emission to $0.8 \pm 0.3 \mathrm{AU}$. This value is consistent with most of our derived ring-fit radii (Table 3 ). The high visibility values and small closures phases (which are approximately zero; see Fig. 2) of our observations and of the observations reported by Wheelwright et al. (2013) do not allow us to detect any binary signature. Binarity is believed to be a key property in B[e] stars (Miroshnichenko 2007).

Our geometric ring-fit radii $(\sim 0.8-1.6 \mathrm{AU})$ are approximately 3-5 times smaller than the radius predicted by the sizeluminosity relation ( $4.2 \mathrm{AU}$; Fig. 5). The predicted radii of the size-luminosity relation (Monnier et al. 2005) are based on the assumption that the dust sublimates at a temperature of $1500 \mathrm{~K}$ and the inner cavity is dust-free and optically thin. These assumptions are approximately valid for HAe stars. However, several interferometric observations suggest that some of the more massive HBe stars harbor a gaseous, optically thick disk inside the dust disk. This gas disk can partially shield the stellar radiation, thereby letting dust survive closer to the star (Monnier \& Millan-Gabet 2002; Monnier et al. 2005; Millan-Gabet et al. 2007; Weigelt et al. 2011).

Our best-fit temperature-gradient model (Table 4, Figs. 3, 4) consists of the stellar point source and an extended disk with an inner radius of $r_{\text {in }}=0.67_{-0.21}^{+0.51} \mathrm{AU}$. This inner radius is approximately 1.6 to 2.3 times smaller than the elongated ring-fit radii derived with geometric modeling (Table 3 ) but also corresponds to a higher temperature. We derived a disk inclination of $i=52.6_{-11.0}^{+14.7 \circ}$ and a position angle of the disk semi-major 
A\&A 569, A25 (2014)

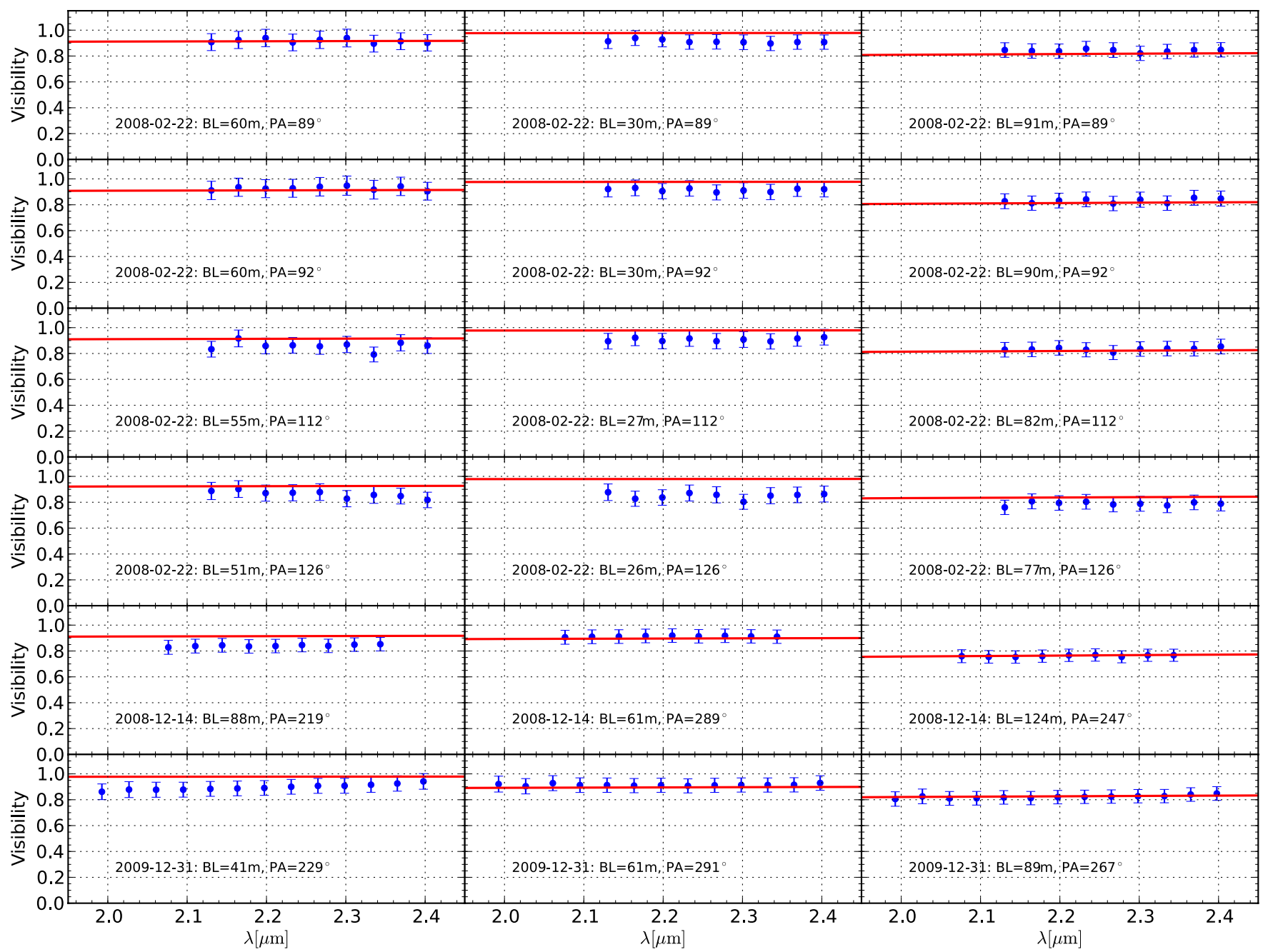

Fig. 3. Comparison of the visibilities of the best-fit temperature-gradient model (red line; see Table 4) with the wavelength-dependent AMBER visibilities (blue dots). The wavelength range of the visibilities is different for all measurements because of different data quality as explained in Sect. 2.
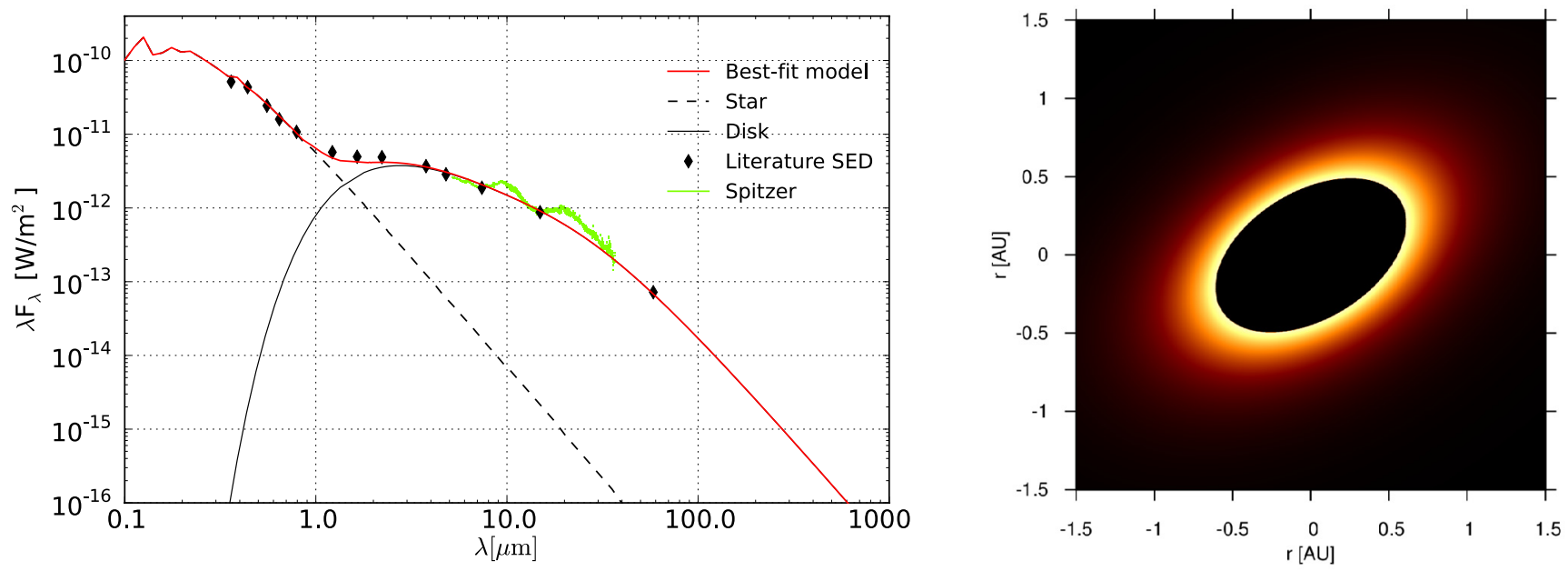

Fig. 4. Left: observed SED (black diamonds) and SED of the best-fit temperature-gradient model (Table 4). The total SED (red line) consists of the stellar flux (Kurucz model, black dashed line) and the flux of the temperature-gradient disk (black solid line). Right: two-dimensional intensity distribution of the best-fit model at $2 \mu \mathrm{m}$. The color-scaling is linear in arbitrary units. The star is not displayed.

axis of $\vartheta=121.4_{-46.8}^{30.6+\circ}$, which are similar to the values derived with geometric modeling, but not very well constrained due to the large error bars of the NIR visibilities (cf. Fig. A.1 in the
Appendix). The derived inner temperature $T_{\text {in }}=2200_{-341}^{+752} \mathrm{~K}$ is too hot for the standard dust composition consisting mainly of silicates, but the existence of refractory dust grains (e.g., iron, 


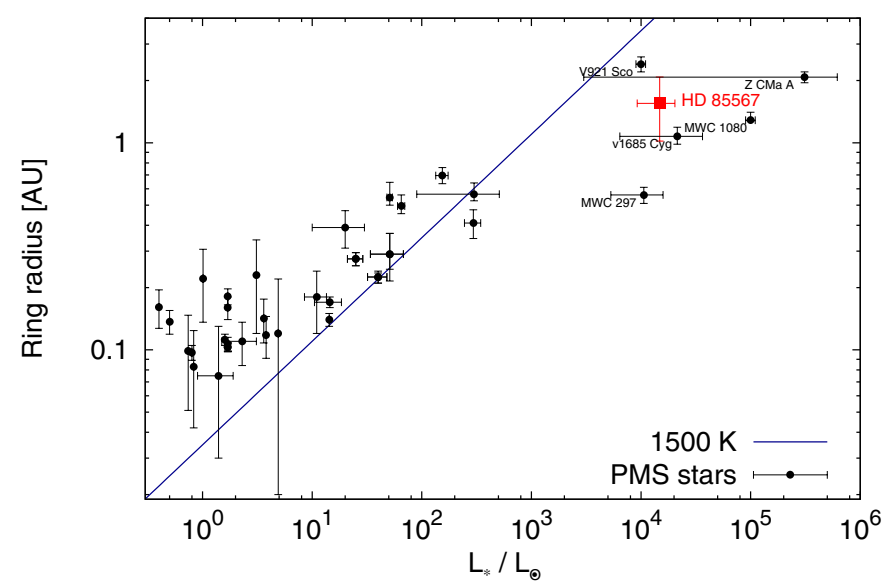

Fig. 5. Position of HD 85567 (red square) in the size-luminosity diagram of $\mathrm{T}$ Tauri stars, Herbig Ae, and Herbig Be stars. The data are taken from the literature (Eisner et al. 2004; Monnier et al. 2005; Kraus et al. 2008a; Pinte et al. 2008; Weigelt et al. 2011; Chen et al. 2012; Kreplin et al. 2012; Vural et al. 2012; Wang et al. 2012; Kreplin et al. 2013; Vural et al. 2014). The solid line shows the theoretical dependence of the inner ring radius on the luminosity for an optically thin disk hole and a dust sublimation temperature of $1500 \mathrm{~K}$ (Monnier et al. 2005).

graphite, corundum; Benisty et al. 2010) may explain this high temperature. Gas emission inside the dust disk can also contribute to the $K$-band visibility and make the average NIR radius appear smaller (e.g., Kraus et al. 2008b). A size estimate based on the accretion rate supports the presence of an optically thick inner disk (Wheelwright et al. 2013). By modeling the CO bandhead emission, these authors found that a compact (inner radius approximately $0.2-1 \mathrm{AU}$ ), optically thick gas disk can reproduce their measurements. This agrees with the hypothesis of shielded stellar radiation, which leads to small dust sublimation radii, which agrees with our observations.

\section{Conclusion}

We used geometric and temperature-gradient models to interpret our VLTI/AMBER data and the SED of the Herbig B[e] star HD 85567. We derived geometric ring models with inner radii of 0.8-1.6 AU, which are approximately 3-5 times smaller than that predicted by the size-luminosity relation. Using temperature-gradient modeling, we found a hot $\left(T_{\text {in }} \sim 2200 \mathrm{~K}\right)$ inner disk rim with a small $\left(r_{\text {in }} \sim 0.67 \mathrm{AU}\right)$ inner radius. The undersized inner disk radius obtained with both geometric and temperature-gradient modeling agrees with measurements of several other $\mathrm{HBe}$ stars and with previous measurements of this object. The small inner radius provides further support to the existence of an optically thick gaseous inner disk shielding the stellar radiation in Herbig Be stars.

Acknowledgements. This research has made use of the NASA/ IPAC Infrared Science Archive, which is operated by the Jet Propulsion Laboratory, California Institute of Technology, under contract with the National Aeronautics and Space Administration. We thank our ESO colleagues at the Paranal observatory for their excellent collaboration.

\section{References}

Baines, D., Oudmaijer, R. D., Porter, J. M., \& Pozzo, M. 2006, MNRAS, 367, 737

Benisty, M., Natta, A., Isella, A., et al. 2010, A\&A, 511, A74

Chelli, A., Utrera, O. H., \& Duvert, G. 2009, A\&A, 502, 705

Chen, L., Kreplin, A., Wang, Y., et al. 2012, A\&A, 541, A104

Dullemond, C. P., \& Monnier, J. D. 2010, ARA\&A, 48, 205

Dullemond, C. P., Dominik, C., \& Natta, A. 2001, ApJ, 560, 957

Eisner, J. A., Lane, B. F., Akeson, R. L., Hillenbrand, L. A., \& Sargent, A. I. 2003, ApJ, 588, 360

Eisner, J. A., Lane, B. F., Hillenbrand, L. A., Akeson, R. L., \& Sargent, A. I. 2004, ApJ, 613, 1049

Ilee, J. D. 2013, Ph.D. Thesis, University of Leeds

Kraus, S., Hofmann, K.-H., Benisty, M., et al. 2008a, A\&A, 489, 1157

Kraus, S., Preibisch, T., \& Ohnaka, K. 2008b, ApJ, 676, 490

Kraus, S., Hofmann, K.-H., Menten, K. M., et al. 2010, Nature, 466, 339

Kraus, S., Calvet, N., Hartmann, L., et al. 2012, ApJ, 752, 11

Kreplin, A., Kraus, S., Hofmann, K.-H., et al. 2012, A\&A, 537, A103

Kreplin, A., Weigelt, G., Kraus, S., et al. 2013, A\&A, 551, A21

Lafrasse, S., Mella, G., Bonneau, D., et al. 2010, VizieR Online Data Catalog: II/300

Lamers, H. J. G. L. M., Zickgraf, F.-J., de Winter, D., Houziaux, L., \& Zorec, J. 1998, A\&A, 340, 117

Malbet, F., Lachaume, R., Berger, J.-P., et al. 2005, A\&A, 437, 627

Malfait, K., Bogaert, E., \& Waelkens, C. 1998, A\&A, 331, 211

Millan-Gabet, R., Schloerb, F. P., \& Traub, W. A. 2001, ApJ, 546, 358

Millan-Gabet, R., Malbet, F., Akeson, R., et al. 2007, in Protostars and Planets V, 539

Miroshnichenko, A. S. 2007, ApJ, 667, 497

Miroshnichenko, A. S., Levato, H., Bjorkman, K. S., \& Grosso, M. 2001, A\&A, 371,600

Monnier, J. D., \& Millan-Gabet, R. 2002, ApJ, 579, 694

Monnier, J. D., Millan-Gabet, R., Billmeier, R., et al. 2005, ApJ, 624, 832

Natta, A., Prusti, T., Neri, R., et al. 2001, A\&A, 371, 186

Pasinetti Fracassini, L. E., Pastori, L., Covino, S., \& Pozzi, A. 2001, A\&A, 367, 521

Petrov, R. G., Malbet, F., Weigelt, G., et al. 2007, A\&A, 464, 1

Pinte, C., Ménard, F., Berger, J. P., Benisty, M., \& Malbet, F. 2008, ApJ, 673, L63

Tatulli, E., Millour, F., Chelli, A., et al. 2007, A\&A, 464, 29

Verhoeff, A. P., Waters, L. B. F. M., van den Ancker, M. E., et al. 2012, A\&A, 538, A101

Vural, J., Kreplin, A., Kraus, S., et al. 2012, A\&A, 543, A162

Vural, J., Kreplin, A., Kishimoto, M., et al. 2014, A\&A, 564, A118

Wang, Y., Weigelt, G., Kreplin, A., et al. 2012, A\&A, 545, L10

Weigelt, G., Grinin, V. P., Groh, J. H., et al. 2011, A\&A, 527, A103

Wheelwright, H. E., Weigelt, G., Caratti o Garatti, A., \& Garcia Lopez, R. 2013, A\&A, 558, A116 


\section{Appendix A: $\chi^{2}$-maps}
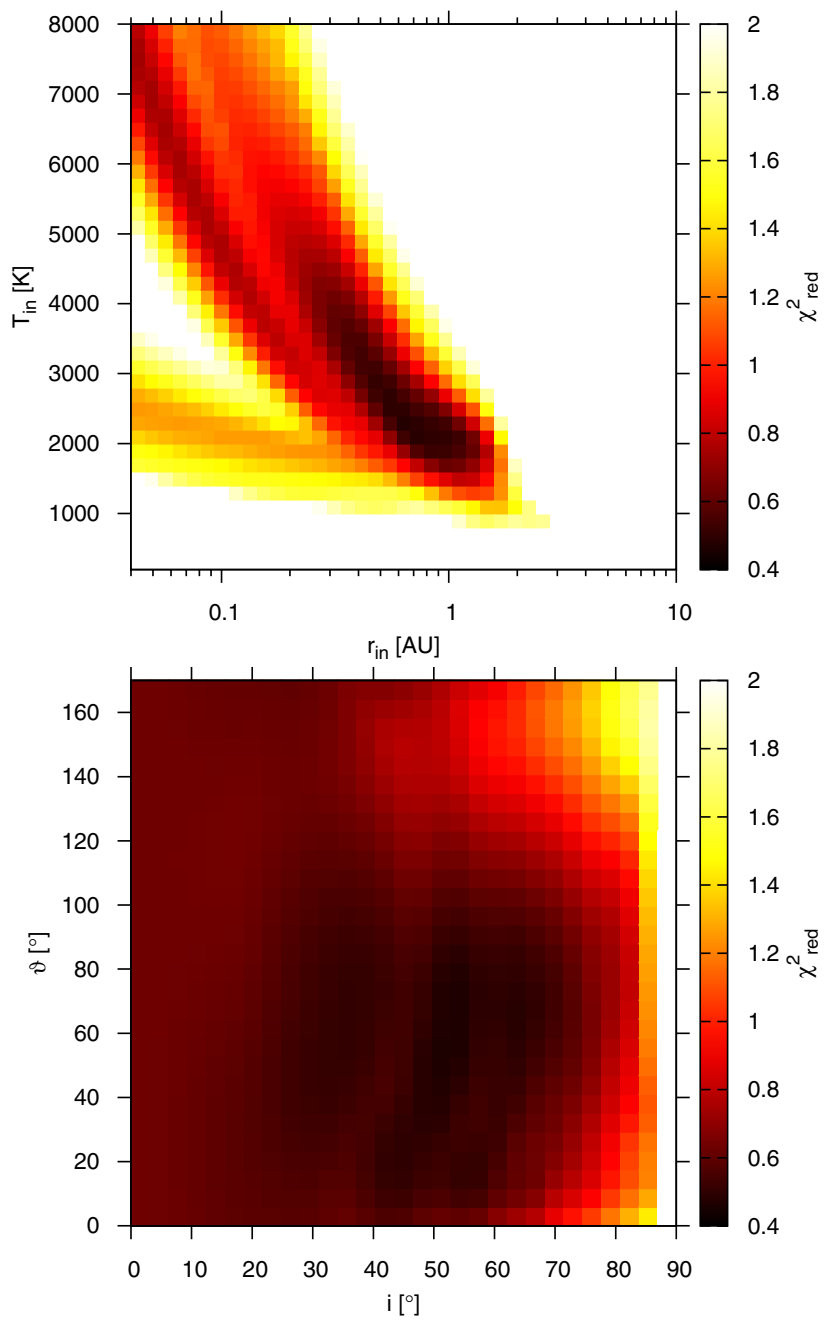

Fig. A.1. Two-dimensional $\chi_{\text {red }}^{2}$ distributions of selected parameters of the temperature-gradient model. 